\title{
Review of: "Detachable dissolvable microneedles: intra-epidermal and intradermal diffusion, effect on skin surface, and application in hyperpigmentation treatment"
}

Peng Xue ${ }^{1}$

1 Southwest University in Chongqing

Potential competing interests: The author(s) declared that no potential competing interests exist.

Sawutdeechaikul et al. report a detachable and degradable microneedle patch for transdermal drug delivery applications. The uniqueness is that they apply this patch on the skin of human volunteers, and the relationship between the time that a conventional dissolvable microneedle array is left on skin without needle detachment from the base is systemically demonstrated. In my opinion, this study may provoke more microneedle designs toward practical applications. with clinical effects and compliance. 